\title{
Influence de la ration sur le taux azoté du pool microbien ruminal
}

\author{
P Lescoat ${ }^{1}, \mathrm{H}$ Archimède ${ }^{1}$, LS Munoz ${ }^{1}, \mathrm{~W}$ Yang ${ }^{2}, \mathrm{C}$ Poncet ${ }^{2}$ \\ 1 INRA, station de nutrition et alimentation de I'INA-PG, 16, rue Claude-Bernard, \\ 75231 Paris Cedex 05; \\ 2 INRA, station de recherches sur la nutrition des herbivores, Theix, \\ 63122 Saint-Genès-Champanelle, France
}

\begin{abstract}
Les systèmes d'expression de la valeur azotée des aliments pour les ruminants supposent que la teneur en azote des microorganismes du rumen (NM) est constante alors qu'elle varie de $4,83 \%$ MS à $10,58 \%$ MS (Clark et al, 1992). Différents facteurs (proportion de concentré, temps après le repas, ...) sont susceptibles d'expliquer en partie ces variations qui semblent notamment refléter l'importance du stockage glucidique microbien. L'objectif de cette étude est de rechercher l'existence de relations entre des caractéristiques de la ration et le NM.
\end{abstract}

Soixante et une données de la bibliographie (10 références) ou de la station (Archimède et al, résultats non publiés) ont été réunies. Le NM moyen est de $7,16 \%$ MS avec un écart type de 1,24\% MS. Les analyses statistiques (tableau I) ont porté sur 3 regroupements : i) les microorganismes libres (BL, $n=32$ ) provenant d'échantillons du fluide ruminal ; ii) les microorganismes libres et attachés $(B A+B L$, $n=29$ ) correspondant à des expériences où un prélèvement des phases liquides et solides, en séparant ou non les microorganismes, a été fait ; et iii) l'ensemble des observations (BAL, $n=61$ ). Le faible nombre d'observations sur les bactéries attachées a empéché de pratiquer des analyses statistiques sur cette population.

L'écart type inter-expériences a été compris entre 0,056 et $1,29 \%$ NM. Ces valeurs traduisent le poids important des conditions propres à chaque étude. En tenant compte de l'influence de l'origine expérimentale (XP, $11 \mathrm{dd}$ ), des relations significatives $(P<0,10)$ apparaissent pour BL et BAL mais pas pour $B A+B L$. L'augmentation du pourcentage de concentré $(\% \mathrm{C})$ de la ration réduit la teneur en NM de l'ensemble des microorganismes (BAL, $P<0,04$ ) et des bactéries libres (BL1, $P<0,02)$. Une augmentation de l'apport des glucides facilement dégradables pourrait expliquer en partie la baisse du NM par accroissement du niveau moyen des réserves intracellulaires. Le pourcentage du NDF dans la ration tend à accroître le NM des bactéries libres (BL2, $P<0,08$ ) tandis que le pourcentage de glucides non structuraux (NSC $=$ MO-MAT-NDF) le diminue $(B L 3, P<0,07)$. Ces résultats appuient l'hypothèse évoquée sur l'effet du concentré ; ils indiquent que, par une substitution glucides-protéines microbiennes, un accroissement de $10 \%$ MS des contenus cellulaires du régime augmenterait de l'ordre de 0,8\% la teneur en glucides de réserves des bactéries. Enfin l'influence du rapport NCS/N ration sur le NM (modèle $B L 4, P<0,07$ ) va dans le même sens.

En conclusion, la composition de la ration influence celle des microorganismes de la panse. Cependant les relations obtenues restent insuffisamment précises pour être intégrées dans des modèles de prévision de la composition de la biomasse microbienne.

Clark JH, Klusmeyer TH, Cameron MR (1992) J Dairy Sci 75, 2304-2323

Tableau I. Modèles explicatifs du taux azoté des microorganismes.

\begin{tabular}{llllll}
\hline Nom & $n$ & Modèle & ETR & $\mathrm{R}^{2}$ & $P$ \\
\hline BAL & 61 & $\left(\right.$ XP(11d)) $-0,00549(0,0025)^{*} \% \mathrm{C}$ & 0,43 & 0,9 & 0,04 \\
BL1 & 32 & $\left(\right.$ XP(6d) $-0,01(0,004)^{*} \% \mathrm{C}$ & 0,48 & 0,8 & 0,02 \\
BL2 & 23 & $\left(\right.$ XP(4d)) $+0,011(0,006)^{*} \%$ NDF & 0,25 & 0,9 & 0,08 \\
BL3 & 23 & $\left(\right.$ XP(4d)) $-0,013(0,006)^{*} \%$ NSC & 0,25 & 0,9 & 0,07 \\
BL4 & 23 & (XP(4d)) - $0,14(0,07)^{*}$ NSC/NR & 0,25 & 0,9 & 0,07 \\
\hline
\end{tabular}

